\title{
INTEGRATING TOPOGRAPHICAL AND TOPOLOGICAL DATA IN THE ESTIMATION OF THE ACTUAL TRAFFIC SITUATION ON AIRPORTS
}

\author{
Christoph MEIER
}

\section{Introduction}

The main necessary functions to manage the traffic on the movement area and the airspace around an airport are surveillance, control, routing and guidance. ${ }^{1}$ Routing is often also termed planning. Today these functions are carried out manually by the controller in the tower and in the apron. But automatic assistance is becoming more and more important with increase of traffic flow in order to reduce the controllers' workload from routine work. The basis for assisting systems is normally a system to estimate the actual traffic situation.

The task of assessing the traffic situation is done today mainly by means of visual observation. The second information source is the voice communication between pilots and controllers. The pilot says where he actually is. Technical systems that help the controller are TV cameras looking at areas not directly seen from the controller, approach radar (Airport Surveillance Radar ASR) and airport radar (Surface Movement Radar SMR). These sources present their information in various ways visual (TV), aural (Voice), from different points of view (all), with clutter (SMR) and synthetic (ASR). The controller fuses all this information in his head in order to estimate the actual traffic situation.

\section{Concept for an automatic airport surveillance}

In a future system the task of traffic situation estimation could be performed by an automatic system. As the traffic objects are partly non-cooperative and partly cooperative also two types of sensors have to be used. Non-cooperative sensors are necessary to detect obstacles and vehicles not equipped with transponders. Cooperative sensors are necessary to identify objects. In order to present an 
unambiguous traffic situation on a synthetic display to the controller a data fusion process is necessary to fuse at least the information of these two sensor types. But also non-sensor information should be taken into account as presented in Figure 1.

Internal sensors are those installed for traffic situation assessment. Examples for sensors actually under development are:

- SMR with digital target extraction

- Near range Radar Network NRN ${ }^{2}$

- TV or infrared cameras with image processing

- $\quad$ SSR Mode S multilateration ${ }^{3}$

- D-GPS with automatic downlink

- Fiber Optical Sensor FOS ${ }^{4}$

- Aircraft Registration Mark Identification ${ }^{5}$

Of cause further sensors are possible. When such a multi sensor system is applied to an airport the types and locations of the sensors have to be selected according to the needs of the airport in order to optimize the cost / benefit ratio.

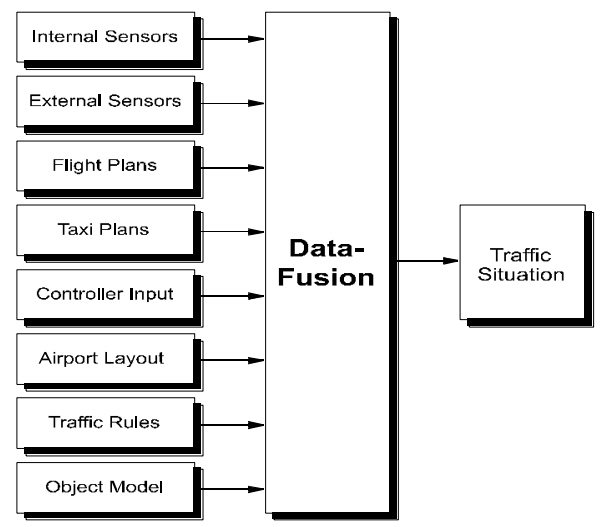

\section{Figure 1: Data Fusion Concept}

External sensors are those already installed for different purposes. Often an ASR for airspace surveillance around the airport is available. External sensors as well as flight plans give valuable hints on expected traffic objects. This information can be used, e.g., for identification purposes. Taxi plans might be available from an automatic routing system. They define with a certain probability the future route and future actions of a traffic object on the airport. Controller inputs such as guidance instructions also define the future behavior of traffic objects. The use of this 
information would require reliable speech recognition. More realistic is the use of controller inputs concerning the object identity (optional manual labeling). The airport layout defines in most cases all possible routes a traffic object can take on an airport. Heavy aircraft are not able to taxi beside the taxiways. Furthermore, traffic rules limit the number of probable routes for a traffic object. Finally a physical object model is of course used to integrate the knowledge that traffic objects do not jump from one place to another.

The advantage of integrating this a priori information is that it can be quite easily obtained and that it requires little maintenance. The problem of integrating such assumptions, i.e., that traffic objects use exclusively the taxiways or that they respect all traffic rules all the time, includes the risk that the traffic situation estimation could fail if these assumptions are violated. So, methods have to be developed and used that cope with these risks.

\section{Modeling the airport layout}

\subsection{Purpose of the model}

A model is always a simplified representation of the main characteristics needed by the user of the model. In this case the data fusion process is the user of the model and therefore defines the requirements to this model. Before modeling the airport layout it has to be defined what tasks the data fusion process has to perform using this model.

1. Positional sensor information normally refers to $x-y$ coordinates. If the data fusion wants to use the airport layout, it has to find out which part of the airport is actually used by a traffic object. This is the mapping from mathematical $2 \mathrm{D}$ (or even 3D) space to a part of the airport.

2. The inverse conclusion from the usage of a certain part of the airport to mathematical coordinates is necessary at least for output purposes.

3. Inference on the probable future behavior of a traffic object derived from the type of the airport part the object is using might also be useful (e.g. high accelerations are probable on runways).

4. It should be possible to conclude from the usage of a part of the airport to the usage of further parts of the airports. This is a prerequisite to find possible routes on the airport.

\subsection{Modeling approach}

The airport layout is modeled in two parts:

- topography 
- topology

The topography describes the physical location of segments of the airport. Especially the boundaries or shapes of these segments are part of the topography. Shapes are defined as sets of topographical points. The definition of these shapes is done in such a way that each point of the mathematical 2D space belongs exactly to one topographical shape. Therefore the shapes are defined mutually exclusive, they do not overlap as shown in figure 2 . The segments are carrying references to the topological elements of the airport.

Topological elements are nodes and links. Nodes are referencing also topographical points - each node is associated with one physical point. This point is normally defined on the edge of a topographical shape. The only exceptions are the ends of taxiways in the apron area. Links are connections between two nodes. Normally exactly one link is referencing a topographical shape. The exceptions are junctions of taxiways where several links reference the same topographical shape. Links are wearing additional attributes to define the operational meaning of that part of the airport, used types are „Runway“, „Taxiway“, „Apron“, „PushBackGate“, „DriveThroughGate“, ApproachArea“, „Grass“, „Hangar“, „Terminal“ and „Street“. Further attributes describe whether the nodes are connected in a straight line or with an arc.

Figure 2 shows the defined shapes, nodes, links and attributes for a small part of an airport. With this model the statement „traffic object at position $\mathrm{P}_{1}$ “ can be unambiguously translated into the statement "traffic object inside shape $\mathrm{S}_{1}$ “ and further into „traffic object following link $\mathrm{L}_{1}$ “ or further into „traffic object on runway $27 R^{\prime \prime}$.

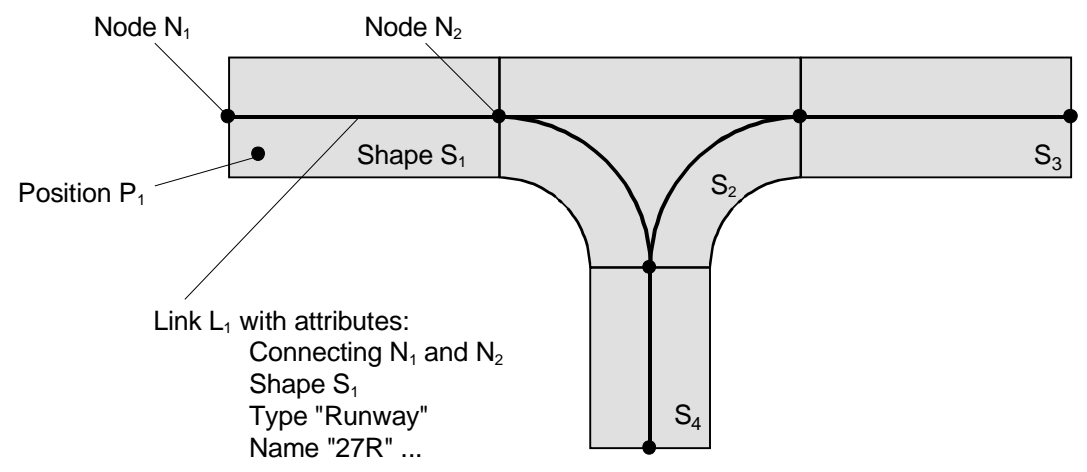

Figure 2: Model of a runway exit 
This translation will only be reliable, if the position is not uncertain. If the traffic objects position $\mathrm{P}_{1}$ has an error of e.g. $10 \mathrm{~m}$, it would be possible that a wrong shape is selected and that all further conclusions based on this shape assumption become also wrong. Furthermore, the conclusion from one single position $\mathrm{P}_{1}$ to the statement „traffic object following link $\mathrm{L}_{1}$ “ is very fragile, the object might just cross the runway. These aspects have to be taken into account when using the model in the state estimation process.

\subsection{Application to a specific airport}

There are several ways to obtain the described airport model. For airports where no reliable maps are available the best approach might be an analysis of satellite images. Another way is the digitalization of paper maps that is very time consuming. But in most cases larger airports today have already an airport map in electronic form. This can be further processed by a CAD system. The processing steps in this case are:

1. Check the validity of the map data, do not trust anything

2. Pick up the relevant topographical points on edges of taxi- and runways, centerlines etc.

3. Construct further points, e.g. centerpoints for arcs

4. Define shapes connecting topographical points

5. Declare some topographical points to be also topological nodes

6. Connect the topological nodes with straight and arc links

7. Give attributes to the links

8. Let the topological elements reference the topographical ones

\section{Using the airport model in the state estimation process}

\subsection{Searching a topographical shape}

When a new traffic object becomes tracked, it is normally not possible to decide immediately which part of the airport it is using. A search phase is necessary. At least one should take into account the direction of movement - the object might just cross a topographical shape - and perhaps the type of the object - a car cannot be on final approach. Because the observation process in the sensors are normally subject to additive noise a soft decision method should be used to find the correct topographical shape. For example, numerical integration of the assumed position error distribution in the limits of the considered shape gives a probability that the object is actually within the shape. Doing this - in the worst case with all shapes - one gets a discrete 
probability distribution on some shapes. To filter this over time a Bayesian framework can be used. The requirement that the shapes must be mutually exclusive avoids the necessity to apply Dempster-Shafers evidence theory. In the search phase a standard Kalman filter - state vector $\mathrm{x}, \mathrm{y}$, vx, vy and additive white gaussian process noise - is used to filter the kinematic sensor data in world coordinates. The search phase is only terminated if a topographical shape is found with a certain high probability or the object leaves the surveillance area.

\subsection{Following a route}

If a topographical shape is found, the conclusion to the used link is done. The link is extended by adjacent links in both directions until a junction or a leaf of the nodelink-network is found. The sequence of links now represents an assumed route the object is following. Another Kalman filter is created filtering the kinematic sensor data in route coordinates. $\mathrm{X}$ represents the progress on the route, $\mathrm{y}$ the deviation to the left side, vx the speed along the route and vy the speed across the route. The Kalman filter from the search phase is not destroyed, it represents the hypothesis that the object is not following a route. So two Kalman filters run in parallel. To avoid divergence of their state estimates a Interacting Multiple Model (IMM) algorithm is used to make continuously a soft decision between the two hypotheses - using the network or not. At junctions further Kalman filters, each representing a feasible route, are created and integrated into the IMM. Route hypotheses are generated when they become possible and are destroyed when they become implausible. Too large deviations from the route make them implausible, the same applies to too high curve accelerations. The first Kalman filter is never destroyed in order to cope with situations where a traffic object leaves the taxiway.

\subsection{Experiments}

To demonstrate the behavior of the proposed method it is tested with simulated sensor data as follows. An object is moving at a constant speed of $10 \mathrm{~m} / \mathrm{s}$ on the airport shown in figure 3 (Braunschweig airport). It starts on the DLR apron and taxies via $\mathrm{F}$ and $\mathrm{C}$. Two fictive sensors observe the moving object. They only deliver positional sensor plots. The characteristics of the two sensors are:

\begin{tabular}{|c|c|c|}
\cline { 2 - 3 } \multicolumn{1}{c|}{} & Sensor 1 & Sensor 2 \\
\hline accuracy in x $(1 \sigma)$ & $15 \mathrm{~m}$ & $3 \mathrm{~m}$ \\
\hline accuracy in y $(1 \sigma)$ & $3 \mathrm{~m}$ & $15 \mathrm{~m}$ \\
\hline update interval & $1,25 \mathrm{sec}$ & $0,95 \mathrm{sec}$ \\
\hline
\end{tabular}




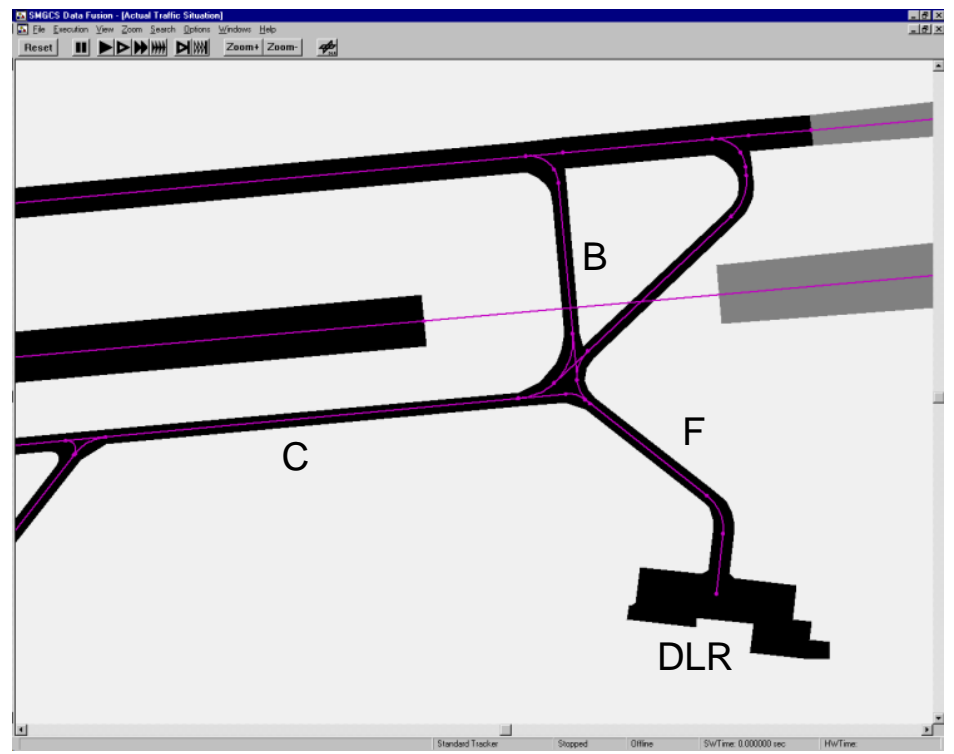

Figure 3: Layout of the Braunschweig airport

First a standard Kalman filter is tested for comparison purposes. The state vector is composed of $\mathrm{x}, \mathrm{y}, \mathrm{vx}$ and vy. Random accelerations in $\mathrm{x}$ and $\mathrm{y}$ direction with a $1 \sigma$ value of $0,5 \mathrm{~m} / \mathrm{s}^{2}$ represent the process noise. Figure 4 shows the result. The gray line represents the true object position. The gray crosses represent the plots of the two sensors and the black solid line the estimates of the Kalman filter.

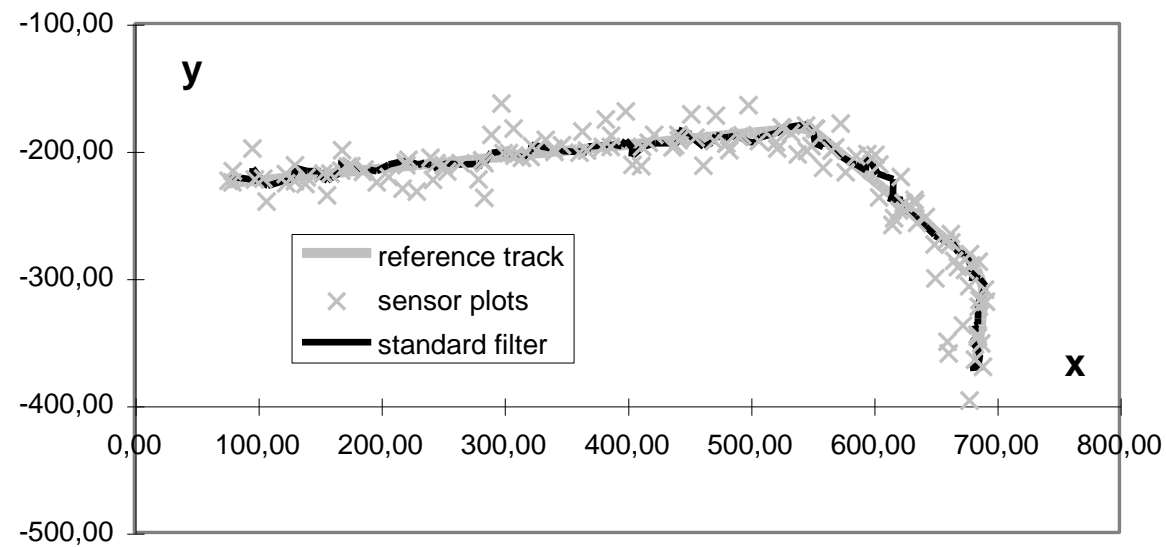

Figure 4: Filtering with a standard Kalman filter in world coordinates 
Figure 5 shows that an improvement can be achieved when filtering in route coordinates instead of world coordinates. That requires the knowledge of the correct route. Figure 6 presents what can happen in the worst case, if the route assumption is wrong (DLR-F-B) - the filter diverges!

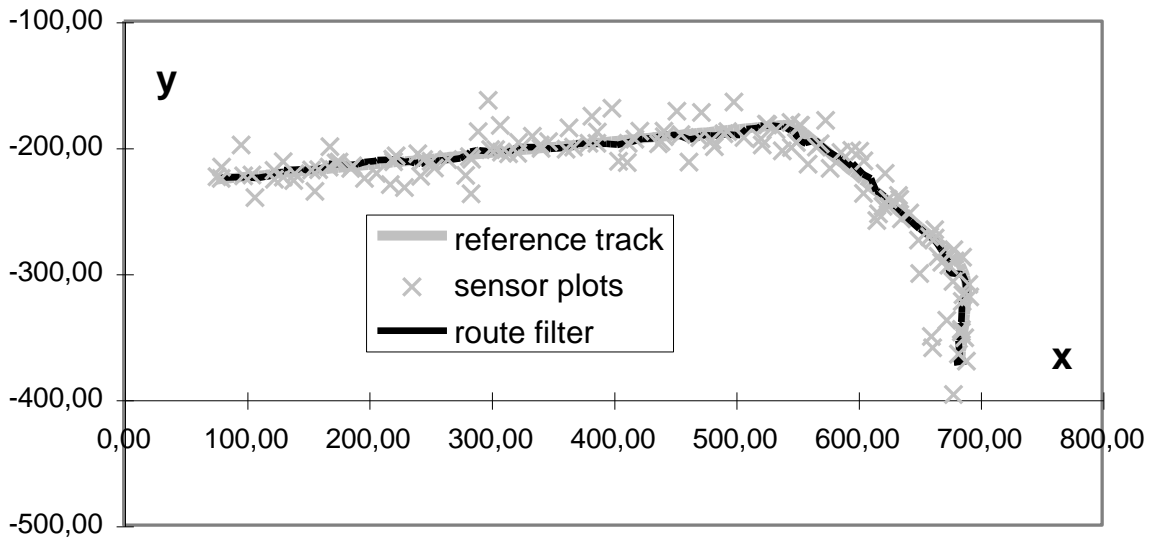

Figure 5: Filtering in route coordinates, assuming the correct route (DLR-F-C)

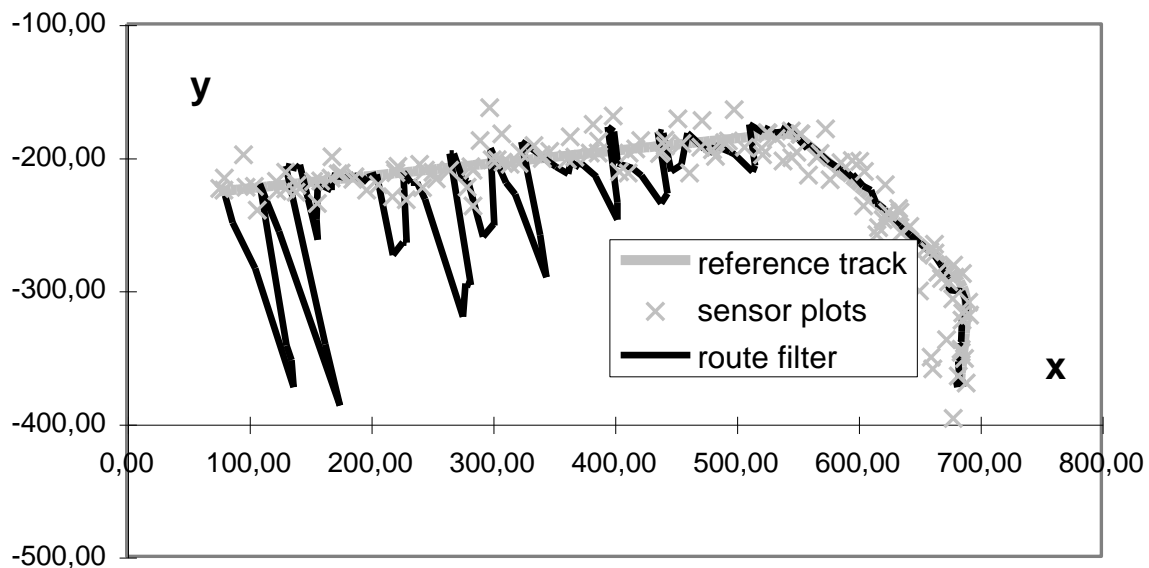

Figure 6: Filtering in route coordinates, assuming the wrong route (DLR-F-B) 
Finally, figure 7 shows the filtering with the proposed IMM method that searches and maintains the correct route automatically.

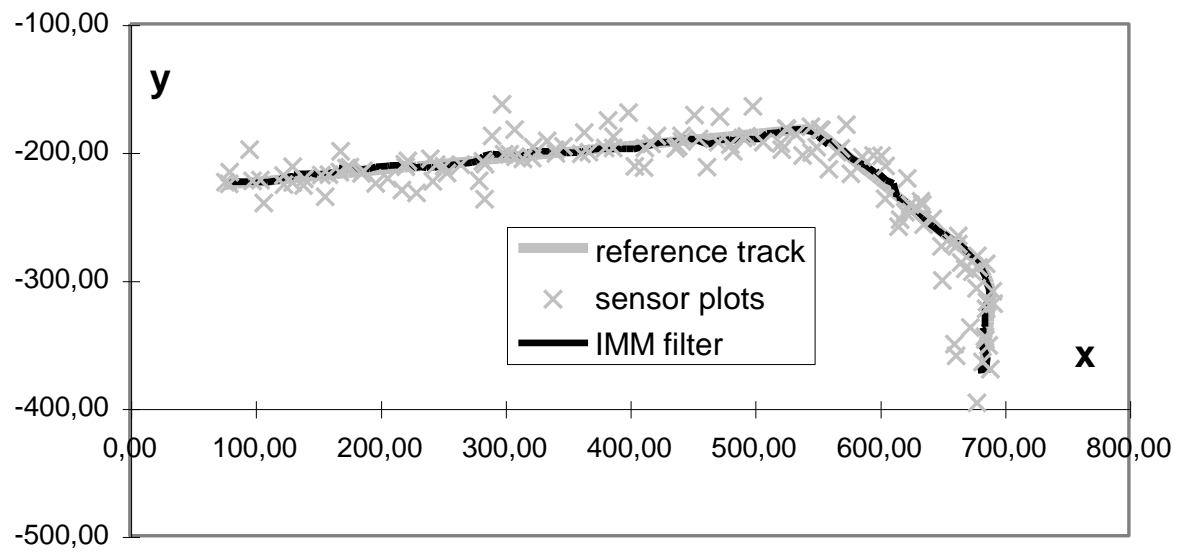

Figure 7: Filtering with the proposed IMM method

The main advantages of the proposed method are:

- The filter predicts the object motion ,around the corner“. Therefore, less systematical errors occur when the object is following a curve. This might also be useful for a data association process to enhance the track continuity.

- The relationship of the traffic object to the airport is permanently estimated. This is part of the data fusion function ,situation assessment“. Following subsystems, e.g., Routing, require such higher level data representation.

The disadvantage is a higher computational complexity. Three Kalman filters integrated in one IMM are approximately as expensive as four stand alone Kalman filters.

\section{Summary}

A method to model and to integrate the airport topography and topology into the traffic situation estimation process has been presented. It performs better than standard Kalman filter solutions. A certain abstraction of the state description of a traffic object from mathematical coordinates to higher level functional airport elements is obtained as a positive side effect. The proposed method is computationally more complex than standard solutions. 


\section{References}

1. ICAO-AWOP. Manual of Advanced Surface Movement Guidance and Control Systems (A-SMGCS) Regional Provisions (EUR), Special AOPG meeting on A-SMGCS (Paris, 3.5.11.1997).

2. Bethke, K.-H., B. Röde, and A. Schroth, "The DLR Short Range Radar Network Installed at Braunschweig Airport: First Results," IRS98 DGON/ITG conference (Munich, 1998).

3. Neufeldt, H., "MAGS, A New Sensor for Airport Surveillance Based on SSR Mode S," IRS98 DGON/ITG conference (Munich, 1998).

4. Fürstenau, N., et al., "Fiber-Optic Sensors for Smart Taxiways," in: DLR-Mitteilung 9602 (Köln, 1996).

5. Döhler, H.-U., E. Groll, and P. Hecker, "Automatic Recognition of Aircraft Registration Marks," in: DLR-Mitteilung 96-02 (Köln, 1996).

CHRISTOPH MEIER received his Diploma in Electrical Engineering in 1990 from the Technical University of Braunschweig. Since then he is with the German Aerospace Center DLR. His main research interest are integrated multi sensor systems and data fusion techniques. Since end of 1997 he is managing the CNS part of the DLR project TARMAC (Taxi and Ramp Management and Control). In 1998 he received Ph.D. degree from the Technical University of Braunschweig. Currently he is head of the department Sensor Systems and Data Communication within DLR's Institute of Flight Guidance. Address: Lilienthalplatz 7, D-38108 Braunschweig, Germany. E-mail: christoph.meier@dlr.de 